\title{
Improving Diabetes Outcomes with a Multimodal Intervention for Less Advantaged Adults with Type 2 Diabetes
}

Karen E. Atler ${ }^{1,}{ }^{*}$, Deana Muñoz ${ }^{1}$, Carly Knowles ${ }^{1}$, Angie Noe ${ }^{2}$, Christine A. Fruhauf ${ }^{3}$, Kathryn McGirr ${ }^{4}$, Tracy L. Nelson ${ }^{5,6}$, Arlene A. Schmid ${ }^{1}$

1. Department of Occupational Therapy, Colorado State University, 200 Occupational Therapy Building 800 Oval Drive, Fort Collins, CO, USA; E-Mails: karen.atler@colostate.edu; deana.munoz@colostate.edu; carly.knowles@colostate.edu; arlene.schmid@colostate.edu

2. Training and Yoga with Angie 5513 W. $1^{\text {st }}$ Street, Greeley, CO, USA; E-Mail: basicfitnesstraining@gmail.com

3. Department of Human Development and Family Studies, Colorado State University, Behavioral Sciences Building 417, Fort Collins, CO, USA; E-Mail: christine.fruhauf@colostate.edu

4. Expanded Food and Nutrition Education Program (EFNEP), Department of Food Science and Human Nutrition, Colorado State University, 102 Gifford, Fort Collins, CO, USA; E-Mail: kathryn.mcgirr@colostate.edu

5. Colorado School of Public Health at CSU, Colorado State University, Fort Collins, CO, USA; E-Mail: tracy.nelson@colostate.edu

6. Department of Health and Exercise Science, Colorado State University, Fort Collins, CO, USA

*Correspondence: Karen E. Atler; E-Mail: karen.atler@colostate.edu

Academic Editor: Damian Skrypnik

Special Issue: Metabolic Syndrome

OBM Integrative and Complementary Medicine 2021, volume 6 , issue 4

doi:10.21926/obm.icm.2104043
Received: May 18, 2021

Accepted: October 25, 2021

Published: November 05, 2021

\section{Abstract}

If proper self-management behaviors are not maintained, diabetes may lead to reduced quality of life (QoL), decreased engagement in everyday activities, disabling health complications, and even death. Less advantaged adults with limited income, lower education, and fewer resources find diabetes self-management (DSM) particularly challenging due to

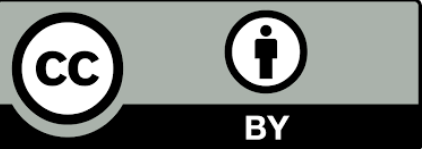

(C) 2021 by the author. This is an open access article distributed under the conditions of the Creative Commons by Attribution License, which permits unrestricted use, distribution, and reproduction in any medium or format, provided the original work is correctly cited. 
healthcare and social inequalities. DSM education is important to understanding diabetesrelated behaviors and improving QoL. There are few evidence informed DSM education programs directed specifically at less advantaged adults. The purpose of this study was to develop and test feasibility and preliminary efficacy of a novel virtual intervention that merged prior evidence-informed yoga, occupational therapy, and nutrition education (MYOT-ED) practices. Five participants were recruited throughout communities near the research university for a nine-week DSM education program that was held twice a week. DSM-related health and QoL measures were tested at baseline, immediately after MY-OT-ED, and six to eight weeks following the intervention. Primary outcomes studied included QoL, DSM, diabetes distress, and diabetes self-efficacy. Percent change was calculated to assess if any significant DSM-related changes occurred. Positive changes were found in all outcomes following the nine-week intervention and were maintained at follow-up. While limitations of the study were noted, results provide preliminary evidence that MY-OT-ED was feasible, acceptable, and has the potential to improve diabetic outcomes for less advantaged adults.

\section{Keywords}

Type 2 diabetes; yoga; occupational therapy; nutrition education; multimodal interventions; diabetes self-management; quality of life; diabetes distress; diabetes self-efficacy

\section{Introduction}

Recent forecasting predicts the worldwide number of people living with diabetes could increase $10 \%$ in the next decade, unless culturally relevant national prevention and intervention programs are created [1]. As a chronic metabolic disorder, type 2 diabetes mellitus (T2DM) may lead to: reduced quality of life (QoL) [2]; reduced engagement in social and community activities [3]; and disabling and costly complications $[4,5]$. Although diabetes education aims to empower people to direct the management of their condition to prevent or decrease complications [6-8], only one-third to one-half of people receiving diabetes education were fully adherent with their health professional's recommendations [9].

Low adherence to diabetes self-management (DSM) can in part be attributed to several psychosocial factors (e.g., social environment and care provider-patient relationships) [10]. As well, people with T2DM report diabetes management can interfere with other daily responsibilities and roles [3], often described as burdensome [11]. Attempts to balance DSM with preferred activities [12], and incorporate DSM into daily routines can negatively impact QoL [2].

Unfortunately, adults with limited income, lower education, and fewer resources find DSM particularly challenging, partially related to health care and social inequities [13]. Additionally, few education opportunities exist for less advantaged adults with diabetes $[14,15]$. This is troubling as these same demographic variables, along with being from a minority racial or ethnic background, are associated with greater risk for diabetes [16], increased complications after diagnosis [17], and higher mortality rates [18]. Therefore, there is considerable need to develop DSM education programs targeting less advantaged adults with T2DM. 
The aim of this pilot study was to examine feasibility of a multimodal approach to educating less advantaged adults with diabetes to integrate DSM into their daily lives. Based on the medical standards of diabetes care, which recommends integration of both physical activity and education into one's lifestyle [6], we merged physical activity (yoga), and habit and lifestyle management (occupational therapy - OT) and nutrition education, into one intervention: Merging Yoga, Occupational Therapy, and nutrition EDucation (MY-OT-ED).

\subsection{Merging Essential Aspects of Diabetes Management}

The American Diabetes Association (ADA) recommends physical activity be integrated into one's lifestyle [6]. Yoga, a form of physical activity, improves blood glucose levels, and reduces common risk factors associated with complications from T2DM [19-21]. Yoga, compared to standard exercise, is believed to address physical and cognitive factors related to poor DSM [21], as well as emotional factors [22]. Other single arm studies examining yoga's impact on adults with diabetes have shown improvements in everyday activities, self-efficacy [23], and QoL [24-26]. However, participants in recent yoga studies expressed the need for additional education and social support to learn to integrate DSM behaviors into daily routines $[23,25]$. This suggests yoga alone is not sufficient to change DSM, as self-management is comprised of a complex set of behaviors that varies greatly between individuals [27].

Occupational therapists, health professionals qualified to work with people with diabetes [11, 28], can play an important role in helping people living with chronic conditions, to develop habits and routines, that promote a healthy lifestyle and address QoL [29]. Developing and modifying habits can help people with chronic conditions sustain behavioral changes beyond the intervention period [30]. There is a unique role for OT to enable individuals with diabetes to integrate DSM into their daily routines and habits [11,31, 32].

In particular, the integration of meal planning and healthy eating into lifestyle changes is essential for management of diabetes [33]. While there is not a one-size-fits-all approach, for example, portion control, combining macronutrients, and tools to support meal planning are essential aspects to healthy eating. One successful nutrition education program available to less advantaged families is the Expanded Food and Nutrition Education Program (EFNEP). This federally funded nutrition education program targets families to influence nutrition and physical activity behaviors but is not specific to T2DM. In 2020, 91\% of Colorado EFNEP participants reported improved diet quality practices and $80 \%$ reported improving their level of physical activity [34].

Currently there are no proven group interventions that prioritize integration of DSM into daily activities and routines while simultaneously including guided physical activity and nutrition education. Our preliminary work includes yoga studies with people with diabetes [23, 25], occupation-focused DSM influenced by best practices [32], and research related to merging yoga and occupational therapy $[35,36]$. Thus, the purpose of this study was to develop and test the feasibility and benefits of a novel multi-modal intervention, the Merging Yoga, Occupational Therapy, and nutrition EDucation (MY-OT-ED) intervention. As a result, our two research questions were: 1 ) is MY-OT-ED feasible, and 2) does MY-OT-ED improve QoL and DSM among an underserved population of less advantaged adults with T2DM? 


\section{Materials and Methods}

\subsection{Design}

The non-control feasibility and longitudinal pilot study of MY-OT-ED included multiple assessments completed before and after the intervention, as well as 6 to 8 weeks post intervention. All aspects of MY-OT-ED were delivered via a secure virtual format due to COVID-19 restrictions. All procedures were approved by the institutional review board.

\subsection{Participants}

Recruitment included online and print media, E-mail, and distribution of flyers to local laundromats, support groups, community centers, food pantries, and health centers. Inclusion criteria included: a) being an adult with type 2 diabetes; b) currently receiving or qualifying for federal food assistance; $c$ ) being able to speak, read, and understand English; and d) having a child or grandchild $\leq 18$ years in the home (secondary to EFNEP federal programming regulations). All participants consented and received $\$ 25$ for each assessment completed (up to $\$ 75.00$ ).

\subsection{Outcome Measures}

Participants completed assessments online, with assistance as needed provided by trained research assistants. Demographic and diabetes characteristics were collected at baseline. Feasibility benchmarks were set a priori, based on our prior studies [35, 36], and included: recruitment; safety; attendance; and participants' ability to complete the intervention. MY-OT-ED was considered feasible if: $30 \%$ of screened participants consented, $\leq 1$ participant experienced a serious adverse event, all participants attended $\geq 50 \%$ of sessions, and $80 \%$ of participants completed the intervention. Following completion of MY-OT-ED, participants completed nine satisfaction questions on a Likert scale of 1 (very dissatisfied) -7 (very satisfied).

All outcome measures were assessed before (T1), after the nine-week intervention (T2), and 6-8 weeks later (T3). We assessed four primary outcomes: QoL, diabetes self-management, diabetes distress, and diabetes self-efficacy.

We assessed QoL using the World Health Organization Quality of Life Brief (WHOQOL-BREF). The WHOQOL-BREF contains 26 items that measure QoL in four domains: physical health, psychological health, social relationships, and environment [37]. The WHOQOL-BREF is a valid and reliable measure for T2DM [38] and higher scores indicate greater QoL, with a possible score of 100 after raw score transformation.

We assessed diabetes self-management using the Diabetes Self-Management Questionnaire (DSMQ) which contains 16 items, including four self-management subscales: glucose management, dietary control, physical activity, and health care usage [39]. The DSMQ is considered a valid and reliable measure [40]. Transformed scores range from 0 to 10; higher scores indicate more suitable self-management behaviors.

We assessed diabetes distress using the Diabetes Distress Scale (DDS) which includes 17-items and produces an overall distress measure and four subscale scores: emotional, physician-related, regimen-related, and interpersonal distress [41]. A mean score of 3 or more in any subscale indicates "moderate distress" and a need for clinical care [42]. Higher DDS scores are associated 
with poorer quality of self-care behaviors, elevated blood lipid levels, and depressive affect $(r>0.33)$ [41].

Last we assessed diabetes self-efficacy with the Stanford Diabetes Self-Efficacy Scale (StanfordDSES) which consists of 8 items with an overall score indicating self-efficacy or confidence in being able to complete DSM activities [43]. The DSES measure is sensitive to note DSE related changes among adults with T2DM [44]. Higher scores indicate higher levels of self-efficacy [45].

\subsection{Intervention}

We developed MY-OT-ED as a standardized group intervention that merged an evidence-based nutrition education class (EFNEP) [45], group occupational therapy, and elements of our prior yoga for with this population [23-26] (Table 1). Materials and incentives (e.g., glucose monitoring supplies, food to try diabetic recipes, and yoga materials) were delivered to participant homes. Additionally, we loaned technology resources (e.g., tablets and Wi-Fi hot spots) to participants as needed. MYOT-ED occurred twice weekly for 9 weeks. The majority of content was delivered synchronously, where the leaders and participants engaged with each other on-line at the same time. For each week, the first session focused on nutrition and the second session addressed OT and yoga.

Table 1 MY-OT-ED session topics organized by week.

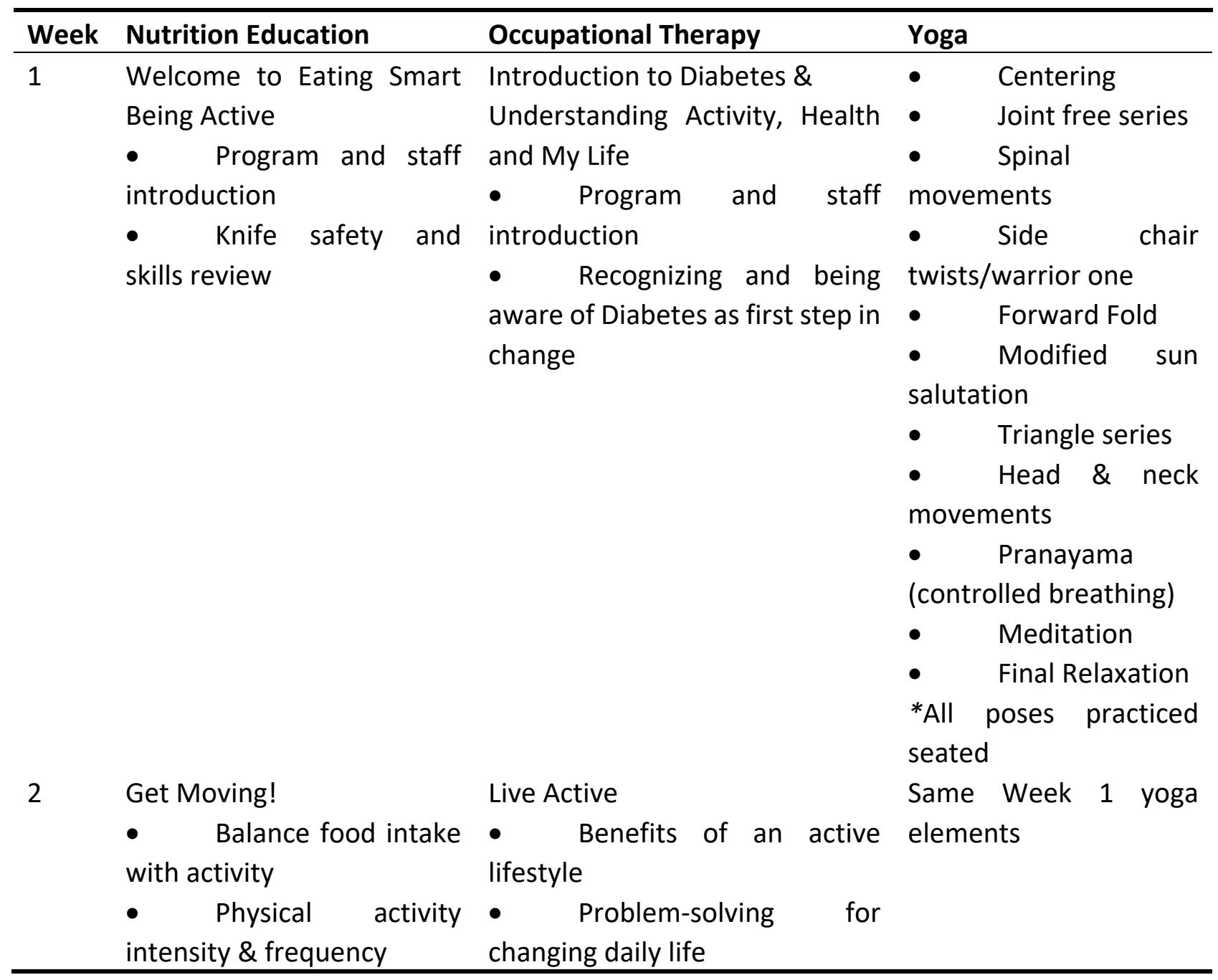




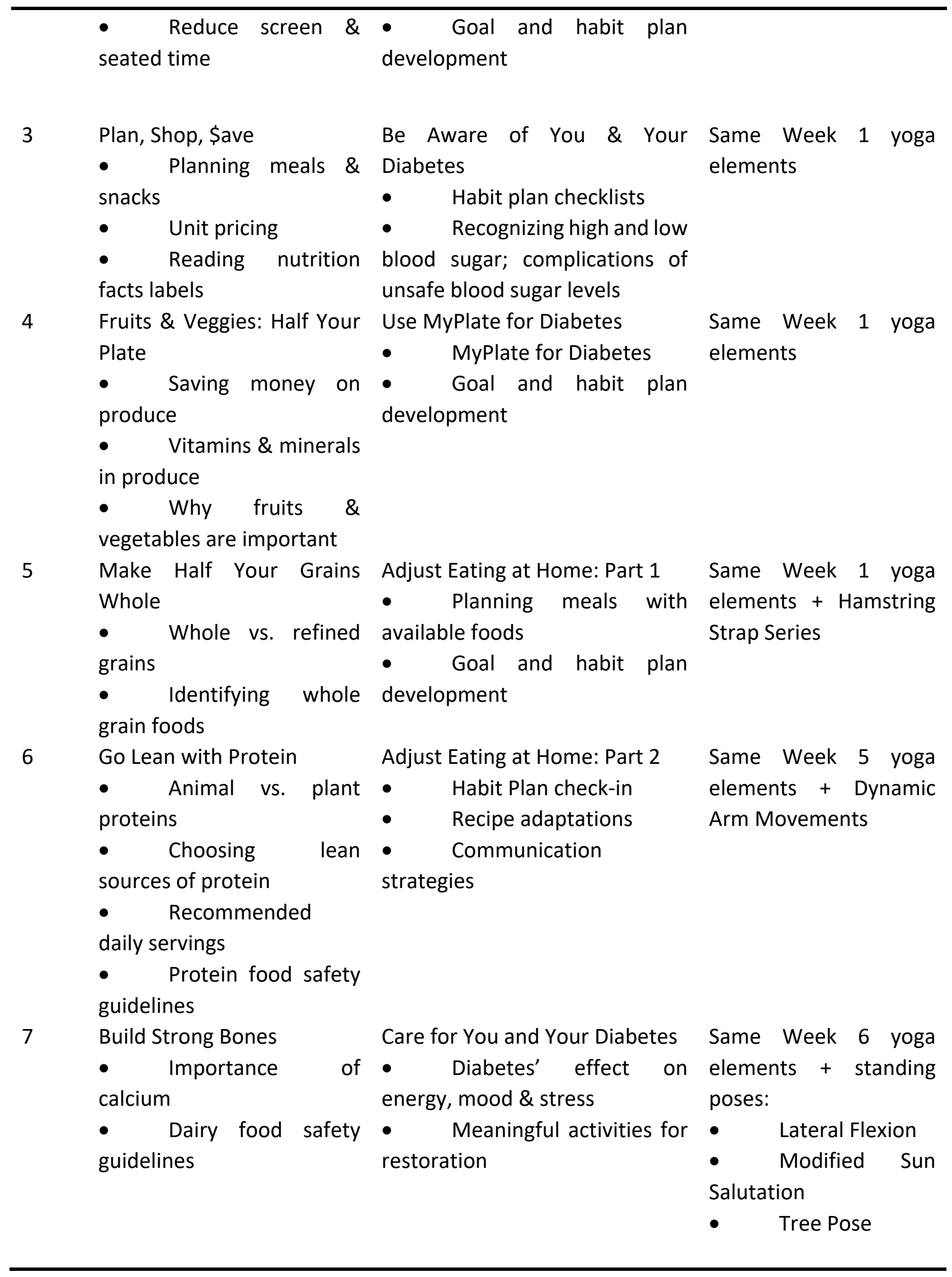




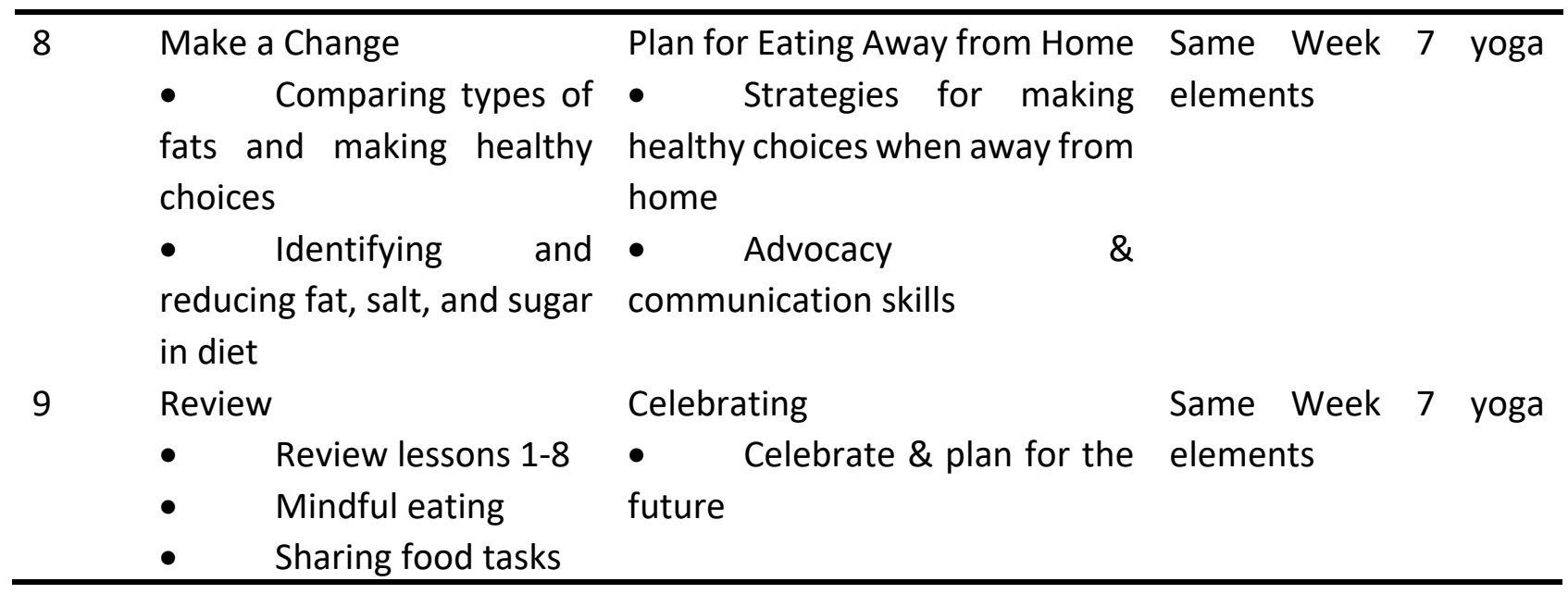

The nutrition program included: online video sessions viewed prior to the weekly synchronous discussions led by the trained EFNEP peer educator. The 30 to 45-minute discussion sessions included topics such as diet quality, physical activity, food resource management, food safety, and food security [45].

OT and yoga were both 50-minute sessions. Group OT, delivered by a licensed occupational therapist, included information on diabetes, its impact on everyday life, and the development of habits and routines to support engagement in DSM while enjoying daily life. We designed sessions following best practice guidelines for the role of occupational therapy in DSM interventions [11, 32] and unpublished descriptive data collected during a pilot study designed to develop 1-to-1 occupation focused DSM education for people with T2DM.

Yoga sessions were delivered by a certified yoga therapist and modified for the T2DM population [23]. Yoga sessions included modified physical postures, breathwork, hand mudras, and meditation. To ensure participant safety, we visually monitored participants and didn't include floor postures. Verbal and visual cues reminded participants to take breaks, focus on breathing and follow individualized accommodations for physical limitations. Each week, participants monitored blood glucose levels before and after yoga.

\subsection{Data Analysis}

Data were analyzed using the Statistical Package for the Social Science 26 [46]. Descriptive statistics were conducted to report demographics, diabetes characteristics, and feasibility data. Due to the small sample size, we calculated percent change for each outcome measure between baseline (T1) and post (T2) assessments (T1-T2/T1x100), and between post (T2) and six to eight week followup (T3) (T2-T3/T2x100).

\section{Results}

\subsection{Participants}

The mean age of the five MY-OT-ED participants was $52.8 \pm 10.6$ years old. Three participants were female individuals; two male individuals. All participants reported being disabled or not working and received either Medicaid or Medicare insurance. See Table 2 for a synthesis of additional demographic and diabetes related information. 
Table 2 Demographic information of MY-OT-ED participants.

\begin{tabular}{ll}
\hline Demographic Measures & $\mathbf{n = 5}$ \\
\hline Time Since T2DM Diagnosis & $1(20 \%)$ \\
$<1$ year & $2(40 \%)$ \\
1-4 years & $2(40 \%)$ \\
4+ years & \\
Marital Status & $3(60 \%)$ \\
Partnered & $2(40 \%)$ \\
Divorced & \\
Ethnicity & $2(40 \%)$ \\
Hispanic/Latinx & $3(60 \%)$ \\
Non-Hispanic/Latinx & \\
Education & $1(20 \%)$ \\
Completed some school & $1(20 \%)$ \\
High School/GED & $1(20 \%)$ \\
Some College & $2(40 \%)$ \\
Graduated College & \\
Family History of Diabetes & $5(100 \%)$ \\
Family History & \\
Secondary Diabetic Complications & \\
Pain & $5(100 \%)$ \\
Chronic Fatigue & $4(80 \%)$ \\
Numbness & $3(60 \%)$ \\
Visual Changes & $3(60 \%)$ \\
Mood Changes & $3(60 \%)$ \\
Monthly Household Income & \\
$\leq \$ 1,000$ & $3(60 \%)$ \\
\$1,100 - 3,000 & $2(40 \%)$ \\
\hline
\end{tabular}

\subsection{Feasibility}

Regarding recruitment, 16 of 22 people screened were eligible (73\%), and 5 agreed to participate (31\% of eligible). Reasons for ineligibility included: pregnancy, no children in the home, or not having a diagnosis of T2DM. Reasons eligible individuals didn't participate included: stress (e.g., children completing school at home, busy schedules, family illness, or lack of childcare), having a daytime job, or not comfortable with or wanting to participate in the online format. Related to safety, no adverse events were reported. Participants attended 78-83\% (7-7.5 sessions) of the nutrition education, occupational therapy, and yoga sessions. Reasons for missing MY-OT-ED sessions included pain, illness, home invasion/property theft, scheduled medical appointments, parenting responsibilities, and internet connectivity issues. All participants completed the intervention; there was no attrition. All five participants agreed or strongly agreed that they were satisfied with all aspects of the intervention. In addition, all participants were "very satisfied" with instructors' knowledge and the intervention's ability to improve their daily lives. All participants were either 
"satisfied" or "very satisfied" with the instructors' abilities to facilitate the weekly sessions. Two participants $(40 \%)$ reported the nutrition and cooking knowledge gained from the intervention were helpful; two participants (40\%) also indicated the focus on exercise habits, walking, and yoga were helpful. Three participants (60\%) indicated that learning from other participants or staff was the most helpful aspect of the intervention.

\subsection{Outcomes Measures}

Overall, outcome measures improved after the MY-OT-ED intervention (see Table 3 and Figures 1-3). All WHOQOL-BREF domains showed improvement. DSMQ Glucose Monitoring and Physical Behavior scores increased while Dietary Control scores decreased (3\%). DSMQ Healthcare Utilization scores decreased (49\%), as one participant decreased emergency room visits. Additionally, Diabetes Self-Efficacy scores positively increased, and Diabetes Distress Scale scores decreased in all subscales, with the exception of Physician Distress. 
Table 3 Change in outcome measure scores over time.

\begin{tabular}{|c|c|c|c|c|c|}
\hline $\begin{array}{l}\text { Outcome Measures } \\
\text { (range: low - high) }\end{array}$ & $\begin{array}{l}T 1 \\
\text { Mean (SD) } \\
n=5\end{array}$ & $\begin{array}{l}\text { T2 } \\
\text { Mean (SD) } \\
n=5\end{array}$ & $\begin{array}{l}\text { \% Change } \\
\mathrm{T} 1-\mathrm{T} 2\end{array}$ & $\begin{array}{l}\text { T3 } \\
\text { Mean (SD) } \\
n=4\end{array}$ & $\begin{array}{l}\text { \% Change } \\
\text { T2 - T3 }\end{array}$ \\
\hline WHOQOL-Physical $(0-100)$ & $20.2(9.9)$ & $61.2(7.1)$ & $\uparrow 203 \%$ & $64.3(15.4)$ & $\uparrow 5.1 \%$ \\
\hline WHOQOL-BREF Psychological $(0-100)$ & $21.4(5.4)$ & $62.6(13.9)$ & 个193\% & $70.3(13.8)$ & $\uparrow 12.3 \%$ \\
\hline WHOQOL-BREF Social $(0-100)$ & $51.4(20.5)$ & $70.0(12.0)$ & $\uparrow 36 \%$ & $75.0(21.0)$ & $\uparrow 7.1 \%$ \\
\hline WHOQOL-BREF Environmental $(0-100)$ & $22.6(6.8)$ & $80.0(5.4)$ & $\uparrow 254 \%$ & $78.5(11.0)$ & $\downarrow 1.9 \%$ \\
\hline DSMQ Glucose Management (0-10) & $6.67(1.2)$ & $6.93(2.0)$ & $\uparrow 4 \%$ & $5.7(2.9)$ & $\downarrow 17.7 \%$ \\
\hline DSMQ Dietary Control (0-10) & $6.17(2.3)$ & $6.0(2.6)$ & $\downarrow 3 \%$ & $7.1(2.2)$ & 个18.3\% \\
\hline DSMQ Physical Activity (0-10) & $3.21(3.1)$ & $4.89(1.5)$ & $\uparrow 52 \%$ & $4.2(2.5)$ & $\downarrow 14.1 \%$ \\
\hline DSMQ Healthcare Usage (0-10) & $7.41(1.5)$ & $3.78(1.7)$ & $\downarrow 49 \%$ & $5.6(2.5)$ & $\uparrow 48.1 \%$ \\
\hline Diabetes Self-Efficacy Mean (0-10) & $6.25(.96)$ & $8.38(1.4)$ & 个34\% & $8.5(1.1)$ & $\uparrow 1.4 \%$ \\
\hline $\begin{array}{l}\text { Diabetes Distress Survey Overall (1-6) } \\
\geq 3=\text { mod distress }\end{array}$ & $3.07(.39)$ & $1.56(.38)$ & $\downarrow 49 \%$ & $1.5(.4)$ & $\downarrow 3.8 \%$ \\
\hline $\begin{array}{l}\text { Diabetes Distress Survey Emotional Burden (1-6) } \\
\geq 3=\text { mod distress }\end{array}$ & $3.20(.68)$ & $1.48(.30)$ & $\downarrow 54 \%$ & $1.5(.5)$ & No Change \\
\hline $\begin{array}{l}\text { Diabetes Distress Survey Regimen Distress (1-6) } \\
\geq 3=\text { mod distress }\end{array}$ & $3.80(1.0)$ & $1.92(.63)$ & $\downarrow 49 \%$ & $1.6(.5)$ & $\downarrow 16.6 \%$ \\
\hline $\begin{array}{l}\text { Diabetes Distress Survey Interpersonal Distress (1- } \\
6)_{L} \geq 3=\text { mod distress }\end{array}$ & $2.67(1.0)$ & $1.0(0)$ & $\downarrow 63 \%$ & $1.0(0)$ & No Change \\
\hline $\begin{array}{l}\text { Diabetes Distress Survey Physician Distress (1-6) } \\
\geq 3=\bmod \text { distress }\end{array}$ & $2.30(1.1)$ & $1.65(1.1)$ & $\downarrow 28 \%$ & $1.7(.9)$ & 个3.0\% \\
\hline \multicolumn{6}{|c|}{ WHOQOL-BREF=World Health Organization's Quality of Life, Field Trial Version } \\
\hline
\end{tabular}




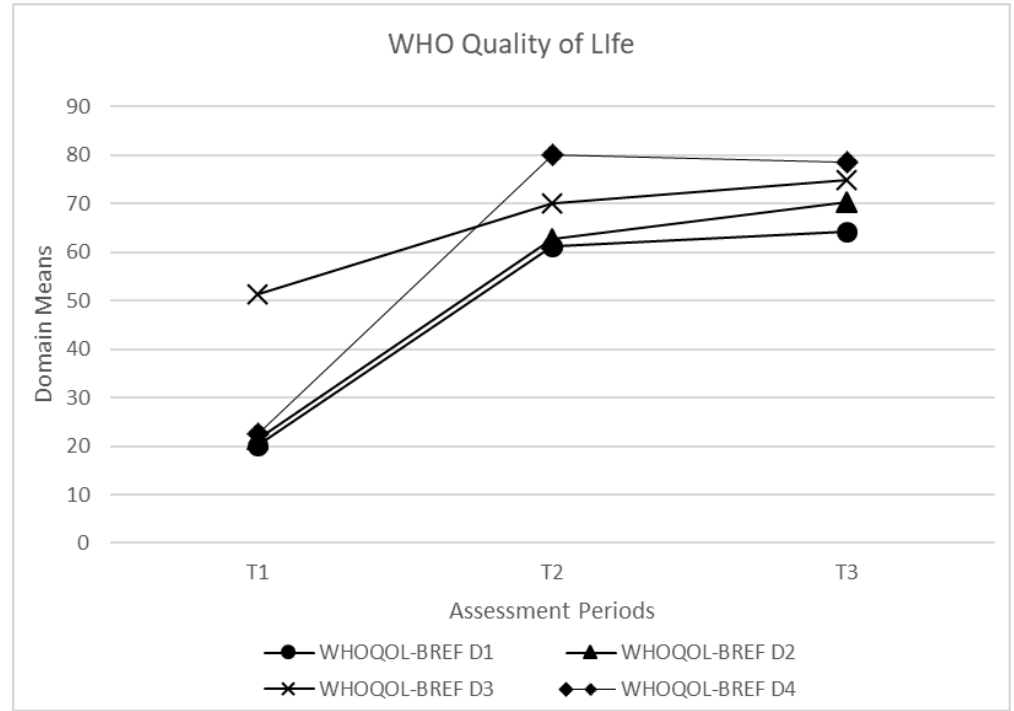

Figure 1 Change in mean scores in QoL over time.

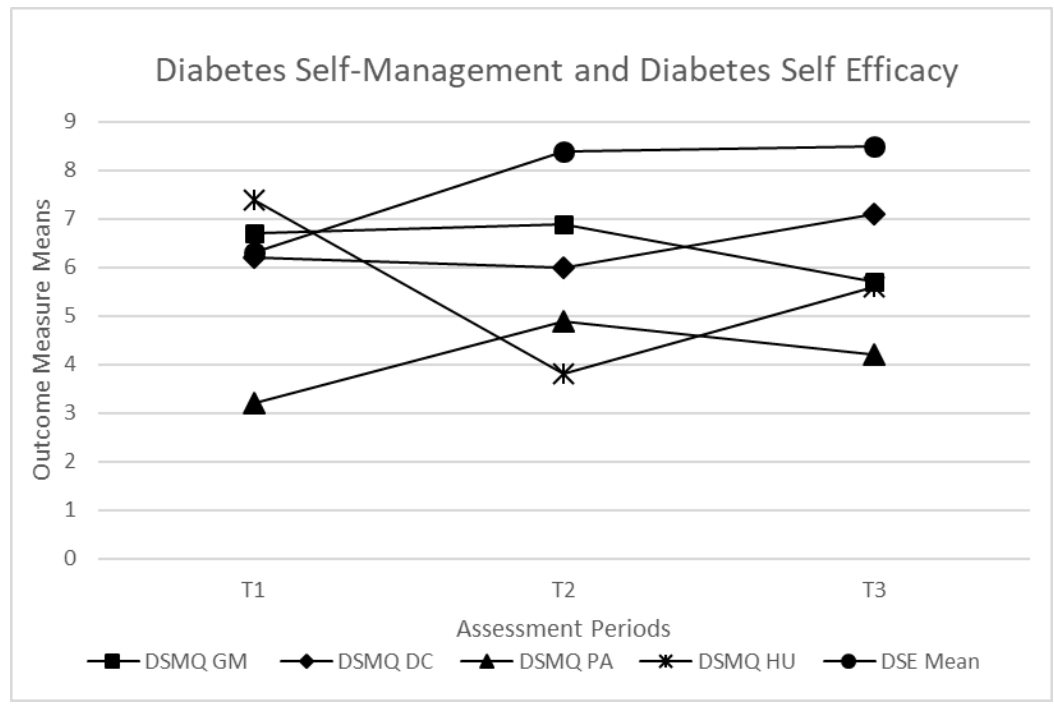

Figure 2 Change in mean scores in DSM and DSE over time.

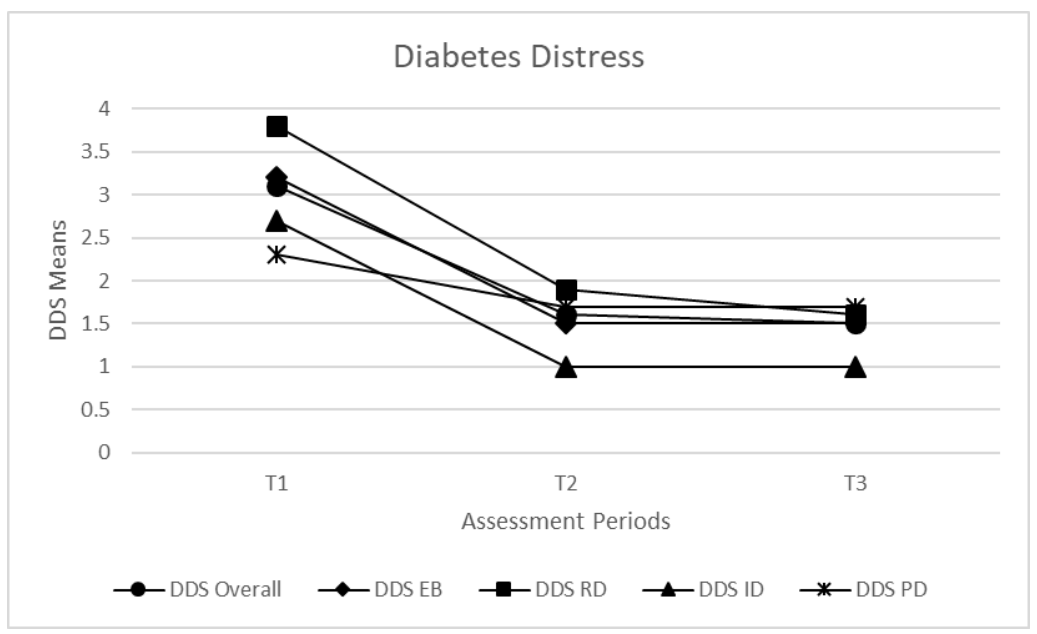

Figure 3 Change in mean scores in DDS over time. 
Four of five participants completed the T3 follow-up assessments. The majority of follow-up (T3) scores either stayed the same or improved when compared to T2 scores, indicating the initial gains made following the nine-week intervention were sustained. Within the DSMQ, glucose management improved, and health care utilization increased.

\section{Discussion}

Results from this feasibility and longitudinal pilot study provide initial evidence that the nineweek multimodal virtual group MY-OT-ED intervention was feasible, acceptable, and potentially beneficial. Our results add to the current evidence that offering group interventions directed at less advantaged adults to develop or establish DSM behaviors can improve their well-being [32]. However, continued development and additional research are required.

\subsection{Feasibility}

The initial examination of feasibility and acceptability of the MY-OT-ED highlighted some important findings as well as areas for continued study. The synchronous virtual intervention was potentially successful and safe to deliver, likely due to blood glucose monitoring before and after yoga, along with modification of yoga postures. However, it was not efficient to merge materials from the general nutrition education program (EFNEP) directly into MY-OT-ED for diabetes. For example, EFNEP education highlights 'MyPlate', the nutrition guide published by the USDA as a central educational tool taught for healthy eating among healthy individuals [45]. In the OT group intervention, the leader employed the 'Plate Method' for diabetes [47, 48] as a central tool to support healthy eating, shopping, and meal planning. In retrospect, use of the Plate Method for Diabetes in the EFNEP education would have simplified learning, reduced confusion, and provided additional time for the integration of the 'Plate Method' as an important tool to help manage blood glucose. Although MY-OT-ED appears feasible, sessions were frequently shortened due to technology and video conferencing access issues. Simplification of the multimodal intervention may address some of these issues, and improve the explicit connections between nutrition education, OT, and yoga. Due to participants feedback that MY-OT-ED needed to be longer, the length and number of sessions requires additional study.

The challenge to recruit more participants for the virtual intervention may be related to the timing of the study during the COVID-19 pandemic. Eligible individuals who choose not to participate expressed they were experiencing many changes and increased stress due to COVID-19 (e.g., children suddenly at home, COVID-related deaths, and limited resources). For future testing of similar multimodal interventions, we recommend removing the EFNP inclusion criteria of having children living in the home, likely allowing more people to participate in and benefit.

Participation in MY-OT-ED exceeded benchmarks established for attendance, and session completion rates. Free and at-home participation may have supported feasibility and accessibility. Perhaps isolation related to COVID-19 quarantines enhanced attendance as participants sought a venue for social interactions. Additionally, participants received multiple weekly reminders and 1:1 virtual technology support. These supports were provided to address commonly expressed concerns and challenges with the use of technology in telehealth services [49]. We recommend continued exploration of ways to use more user-friendly technology and address the instability of internet connections, particularly in rural areas. Development of virtual programming employing 
technology that participants already use daily, such as social media platforms, may be more effective.

More than two-thirds of MY-OT-ED was offered synchronously, which is a less frequently described approach to online delivered DSM education [50]. The results of this study aid in understanding how less advantaged adults felt about a synchronous online format for DSM education. While caution must be taken due to the small sample size, participants' satisfaction with synchronous programming suggests the importance of continued research in this area. Although the virtual delivery resulted in some limitations (i.e., not being able to engage in food preparation during in person EFNEP's interventions), the participants' high satisfaction with being able to learn from others suggests the synchronous sessions supported participants' ability to connect with each other in a supportive environment. Although results were not compared to a control group due to COVID-19 related changes, we recommend a randomized control trial (RCT) trial in order to compare delivery methods, including in-person, virtual, and or combination of in-person and online.

Online yoga for diabetes was feasible and supported accessibility for our participants who may not have had access to formal yoga classes (e.g., time constraints, high cost, lack of inclusion for minority populations). Social and cultural norms around yoga do not always include culturally diverse samples or individuals with disabilities [51]. Therefore, virtual yoga allowed participants to do yoga at home with equipment found around the house and provided through the study. Many MY-OT-ED participants chose incorporating yoga into their daily routines as goals during weeks five through eight. Two participants reported doing some of the yoga postures every day by the end of the study.

\subsection{Outcome Measures}

The results of this study report notable findings related to self-reported improvements in QoL and diabetes self-efficacy and decreases in diabetes distress immediately following completion of MY-OT-ED. While changes reported on the DSMQ were smaller, participants did report a $52 \%$ increase in physical activity behaviors. This may be related to provision of yoga resources in their home, opportunity to engage in weekly virtual yoga, and or 4 out of 5 participants intentionally working on integrating yoga into a habit. The lack of more notable changes in DSM maybe because moving new behaviors into habits requires additional time and practice [30].

Additionally, all five participants reported on the satisfaction survey that participation in MY-OTED positively influenced and changed their lives. Although we do not know what led to the changes in scores, the work by Jiang and colleagues [52] found that self-efficacy was the strongest predictor of DSM and also had a mediating role in the connection between diabetes distress and DSM. We believe that the merging of several interventions providing knowledge, guided physical activity, and opportunities to practice new behaviors with discussion of how to move behaviors into habits may be a critical feature. Several of these aspects are strongly supported by social cognitive theory [53] and are recommended in the national standards for DSM [54].

Due to the ongoing need for DSM education and the challenge of integrating DSM into daily life, development of multimodal interventions that aim to enhance and sustain self-efficacy among less advantaged adults with T2DM remains critical. Current national standards for DSM education do not provide recommendations for length of programs [54], but all participants in this study recommended additional sessions or weeks of the intervention to allow time to integrate the 
knowledge and skills into everyday life. Perhaps future multimodal interventions should also include breaks or booster sessions to promote connection with participants. Continued research is needed to determine the important dosage of multimodal DSM education.

Participants who completed the follow-up assessments $(T 3 ; n=4)$ demonstrated the gains in QoL and diabetes self-efficacy and a reduction in distress were generally maintained six to eight weeks after completion of MY-OT-ED. DSMQ Dietary Control behaviors continued to improve over this time, which is encouraging given the fact the study timeline spanned COVID and year-end holidays. On the other hand, DSMQ Physical Activity behaviors declined, which may speak to the importance of ongoing support recommended to sustain progress made during DSM education [54]. Loss of the group support, which participants frequently mentioned as the intervention ended may be a major factor. Future development and testing will be required to examine the characteristics and format for creating personalized follow-up plans.

\subsection{Limitations and Future Directions}

This study contains several limitations. First, researchers were unable to complete a control group due to COVID-19. Secondly, given the small sample size and geographic location, results are not generalizable. Third, participants received COVID-19 related government-funded relief packages, potentially influencing outcomes related to QoL and distress. Given these findings, larger studies are recommended. Future studies should also compare the outcomes of virtual vs. face-toface or hybrid delivery formats with integration of diabetes specific nutrition education into the OT group.

\section{Conclusion}

Even though COVID-19 required the transition to virtual format, MY-OT-ED appeared to be feasible and potentially efficacious for less advantaged adults with T2DM. Further research is required to incorporate recommendations included in this report, to continue to study the delivery format of DSM education designed for less advantaged adults. Overall, we recommend simplification of the programming and continued focus on strengthening the merger of multimodal interventions for this population, with special attention paid to delivery of culturally relevant interventions. It is essential to continue to create accessible DSM for less advantaged adults to provide them with supports and resources to learn how to incorporate DSM into their daily lives while improving their QoL and ability to enjoy living life.

\section{Acknowledgements}

The authors give sincere thanks to the five participants involved in MY-OT-ED. The authors hope each participant is still living life while managing their diabetes.

\section{Author Contributions}

Karen E. Atler - Principal Investigator of the AOTF grant; designed and implemented the occupational therapy group intervention; assisted with data collection and fidelity checks; authored and edited manuscript. Deana Muñoz - edited occupational therapy intervention; assisted with data collection and fidelity checks; authored and edited manuscript. Carly Knowles - primary data 
collector; edited occupational therapy intervention; assisted with fidelity checks; data analysis; authored and edited manuscript. Angie Noe - designed and implemented yoga therapy intervention; assisted with data collection and fidelity; reviewed and edited manuscript. Christine A. Fruhauf assisted the research team in development of assessments, reviewed and edited manuscript. Kathryn McGirr - co-author of the nutrition education curriculum Eating Smart - Being Active; trained and supervised the EFNEP peer educator; reviewed and edited manuscript. Tracy Nelson assisted research team with study design, reviewed and edited manuscript. Arlene A. Schmid mentor for the Principal Investigator; co-developer of occupational and yoga intervention (MY-OT); authored and edited manuscript.

\section{Funding}

This research was funded by a grant (AOTFIRG19 ATLER) received in 2019 from the American Occupational Therapy Foundation (AOTF).

\section{Competing Interests}

The authors have declared that no competing interests exist.

\section{References}

1. Ampofo AG, Boateng EB. Beyond 2020: Modelling obesity and diabetes prevalence. Diabetes Res Clin Pract. 2020; 167: 108362.

2. Ahlin K, Billhult A. Lifestyle changes - a continuous, inner struggle for women with type 2 diabetes: A qualitative study. Scand J Prim Health Care. 2012; 30: 41-47.

3. Atler KE, Schmid AA, Klinedinst TC, Grimm LA, Marchant TP, Marchant DR, et al. The relationship between quality of life, activity and participation among people with type 2 diabetes mellitus. Occup Ther Health Care. 2018; 32: 341-362.

4. Bailey CJ, Blonde L, Del Prato S, Leiter LA, Nesto R. What are the practical implications for treating diabetes in light of recent evidence? Updated recommendations from the global partnership for effective diabetes management. Diab Vasc Dis Res. 2009; 6: 283-287.

5. World Health Organization. Global report on diabetes. Geneva: World Health Organization; 2016.

6. American Diabetes Association. Lifestyle management: Standards of medical care in diabetes2017. Diabetes Care. 2017; 40: S1-S135.

7. Marrero DG, Ard J, Delamater AM, Peragallo-Dittko V, Mayer-Davis EJ, Nwankwo R, et al. Twenty-first century behavioral medicine: A context for empowering clinicians and patients with diabetes. Diabetes Care. 2013; 36: 463-470.

8. Shrivastava SR, Shrivastava PS, Ramasamy J. Role of self-care in management of diabetes mellitus. J Diabetes Metab Disord. 2013; 12: 1-5.

9. Hirsch IB. The burden of diabetes (Care). Diabetes Care. 2003; 26: 1613-1614.

10. Orvik E, Ribu L, Johansen OE. Spouses' educational needs and perceptions of health in partners with type 2 diabetes. Eur Diabetes Nurs. 2010; 7: 63-69.

11. Pyatak EA. The role of occupational therapy in diabetes self-management interventions. OTJR (Thorofare N J). 2010; 31: 89-96. 
12. Ingadottir B, Halldorsdottir S. To discipline a 'dog': The essential structure of mastering diabetes. Qual Health Res. 2008; 18: 606-619.

13. Hill J, Nielsen M, Fox MH. Understanding the social factors that contribute to diabetes: A means to informing health care and social policies for the chronically ill. Perm J. 2013; 17: 67-72.

14. Shaw K, Killeen M, Sullivan E, Bowman P. Disparities in diabetes self-management education for uninsured and underinsured adults. Diabetes Educ. 2011; 37: 813-819.

15. Gutierrez AP, Fortmann AL, Savin K, Clark TL, Gallo LC. Effectiveness of diabetes selfmanagement education programs for US latinos at improving emotional distress: A systematic review. Diabetes Educ. 2019; 45: 13-33.

16. Assari S, Moghani Lankarani M, Piette JD, Aikens JE. Socioeconomic status and glycemic control in type 2 diabetes; race by gender differences. Healthcare. 2017; 5: 83.

17. Cowie CC. Diabetes diagnosis and control: Missed opportunities to improve health. Diabetes Care. 2019; 42: 994-1004.

18. Saydah SH, Imperatore G, Beckles GL. Socioeconomic status and mortality: Contribution of health care access and psychological distress among U.S. adults with diagnosed diabetes. Diabetes care. 2013; 36: 49-55.

19. Raveendran AV, Deshpandae A, Joshi SR. Therapeutic role of yoga in type 2 diabetes. Endocrinol Metab. 2018; 33: 307-317.

20. Thind H, Guthrie KM, Horowitz S, Conrad M, Bock BC. "I can do almost anything": The experience of adults with type 2 diabetes with a yoga intervention. Complement Ther Clin Pract. 2019; 34: 116-122.

21. Innes KE, Selfe TK. Yoga for adults with type 2 diabetes: A systematic review of controlled trials. J Diabetes Res. 2016; 2016: 6979370.

22. Bock BC, Thind H, Fava JL, Dunsiger S, Guthrie KM, Stroud L, et al. Feasibility of yoga as a complementary therapy for patients with type 2 diabetes: The Healthy Active and in Control (HA1C) study. Complement Ther Med. 2019; 42: 125-131.

23. Willis Boslego LA, Munterfering Phillips CE, Atler KE, Tracy BL, Van Puymbroeck M, Schmid AA. Impact of yoga on balance, balance confidence and occupational performance for adults with diabetic peripheral neuropathy: A pilot study. Br J Occup Ther. 2017; 80: 155-162.

24. Phillips C, Schmid AA, Willis L. Effects of yoga on functional gait and health-related quality of life for adults with Diabetic Peripheral Neuropathy. Am J Occup Ther. 2016: 70; 7011515269p1.

25. Schmid AA, Atler KE, Malcolm MP, Grimm LA, Klinedinst TC, Marchant DR, et al. Yoga improves quality of life and fall risk-factors in a sample of people with chronic pain and type 2 diabetes. Complement Ther Clin Pract. 2018; 31: 369-373.

26. Van Puymbroeck M, Atler K, Portz JD, Schmid AA. Multidimensional improvements in health following hatha yoga for individuals with diabetic peripheral neuropathy. Int J Yoga Therap. 2018; 28: 71-78.

27. Atak N, Gurkan T, Kose K. The effect of education on knowledge, self management behaviours and self efficacy of patients with type 2 diabetes. Aust J Adv Nurs. 2008; 26: 66-74.

28. Williams AS. American Association of daibetes educators disabilities position statement. Diabetes Educ. 2009; 35: 41-44.

29. Gupta J, Chandler B, Toto P. The role of occupational therapy with health promotion. The American Occupational Therapy Association. Available from: https://www.aota.org/- 


\section{/media/Corporate/Files/AboutOT/Professionals/WhatlsOT/HW/Facts/FactSheet HealthProm} otion.pdf.

30. Fritz $\mathrm{H}$, Cutchin MP. Integrating the science of babit: Opportunities for occupational therapy. OTJR (Thorofare N J). 2016; 36: 92-98.

31. Fritz $\mathrm{H}$. The influence of daily routines on engaging in diabetes self-management. Scand J Occup Ther. 2014; 21: 232-240.

32. Pyatak EA, Carandang K, Vigen CL, Blanchard J, Diaz J, Concha-Chavez A, et al. Occupational therapy intervention improves glycemic control and quality of life among young adults with diabetes: The resilient, empowered, active living with diabetes (REAL diabetes) randomized controlled trial. Diabetes Care. 2018: 41: 696-704.

33. Gray A, Threlkeld RJ, Feingold KR, Anawalt B, Boyce A, Chrousos G, et al. Nutritional recommendations for individuals with diabetes. In: Endotext. South Dartmouth, MA: MDText.com, Inc.; 2000.

34. United States Department of Agriculture, National Institute of Food and Agriculture, Expanded Food and Nutrition Education Program. Web-based nutrition education evaluation and reporting system (WebNEERS) [Internet]. 2020. Available from: https://nifa.usda.gov/tool/webneers.

35. Schmid AA, Puymbroeck MV, Portz JD, Atler KE, Fruhauf CA. "Merging yoga and occupational therapy (MY-OT): A feasibility and pilot study". Complement Ther Med. 2016; 28: 44-49.

36. Swink LA, Fling BW, Sharp JL, Fruhauf CA, Atler KE, Schmid AA. Merging yoga and occupational therapy for Parkinson's Disease: A feasibility and pilot program. Occup Ther Health Care. 2020; 34: 351-372.

37. The Whoqol Group. Development of the World Health Organization WHOQOL-BREF quality of life assessment. Psych Med. 1998; 28: 551-558.

38. Reba K, Birhane BW, Gutema H. Validity and reliability of the Amharic version of the World Health Organization's quality of life questionnaire (WHOQOL-BREF) in patients with diagnosed type 2 diabetes in Felege Hiwot Referral Hospital, Ethiopia. J Diabetes Res. 2019; 2019: 3513159.

39. Schmitt A, Gahr A, Hermanns N, Kulzer B, Huber J, Haak T. The Diabetes Self-Management Questionnaire (DSMQ): Development and evaluation of an instrument to assess diabetes selfcare activities associated with glycaemic control. Health Qual Life Outcomes. 2013; 11: 1-14.

40. Schmitt A, Reimer A, Hermanns N, Huber J, Ehrmann D, Schall S, et al. Assessing diabetes selfmanagement with the diabetes self-management questionnaire (DSMQ) can help analyse behavioural problems related to reduced glycaemic control. PLoS One. 2016; 11: e0150774.

41. Polonsky WH, Fisher L, Earles J, Dudl RJ, Lees J, Mullan J, et al. Assessing psychosocial distress in diabetes. Diabetes Care. 2005; 28: 626-631.

42. Fisher L, Glasgow RE, Mullan JT, Skaff MM, Polonsky WH. Development of a brief diabetes distress screening instrument. Ann Fam Med. 2008; 6: 246-252.

43. Harrington C, Carter-Templeton HD, Appel SJ. Diabetes self-management education and selfefficacy among African American women living with type 2 diabetes in rural primary care. J Dr Nurs Pract. 2017; 10: 11-16.

44. Lorig K, Ritter PL, Villa FJ, Armas J. Community-based peer-led diabetes self-management: $A$ randomized trial. Diabetes Educ. 2009; 35: 641-651. 
45. Murray EK, Auld G, Baker SS, Barale K, Franck K, Khan T, et al. Methodology for developing a new EFNEP food and physical activity behaviors questionnaire. J Nutr Educ Behav. 2017; 49: 777-783.e1.

46. Nie NH, Hull CH, Jenkins G, Stienbrenner K, Bent DH. Spss Statistical package for the social sciences. New York: McGraw-Hill Book Co., 1975. pp.41-42.

47. Wansink B, Kranz S. Who's using MyPlate? J Nutr Educ Behav. 2013; 45: 728-732.

48. Raidl M, Spain K, Lanting R, Lockard M, Johnson S, Spencer M, et al. Peer reviewed: The healthy diabetes plate. Prev Chronic Dis. 2007; 4: A12.

49. Lee JY, Chan CK, Chua SS, Paraidathathu T, Lee KK, Tan CS, et al. Using telemedicine to support care for people with type 2 diabetes mellitus: A qualitative analysis of patients' perspectives. BMJ Open. 2019; 9: e026575.

50. Pereira K, Phillips B, Johnson C, Vorderstrasse A. Internet delivered diabetes self-management education: A review. Diabetes Technol Ther. 2015; 17: 55-63.

51. Thomas EV, Warren-Findlow J, Webb JB. Yoga is for every (able) body: A content analysis of disability themes within mainstream yoga media. Int J Yoga. 2019; 12: 68-72.

52. Jiang $X$, Jiang $H$, Li M, Lu Y, Liu K, Sun X. The mediating role of self-efficacy in shaping selfmanagement behaviors among adults with type 2 diabetes. Worldviews Evid Based Nurs. 2019; 16: 151-160.

53. Bandura A. Social foundations of thought and action: A social cognitive theory. Englewood Cliffs, NJ, US: Prentice-Hall, Inc; 1986.

54. Funnell MM, Brown TL, Childs BP, Haas LB, Hosey GM, Jensen B, et al. National standards for diabetes self-management education. Diabetes Care. 2011; 34: S89-S96.

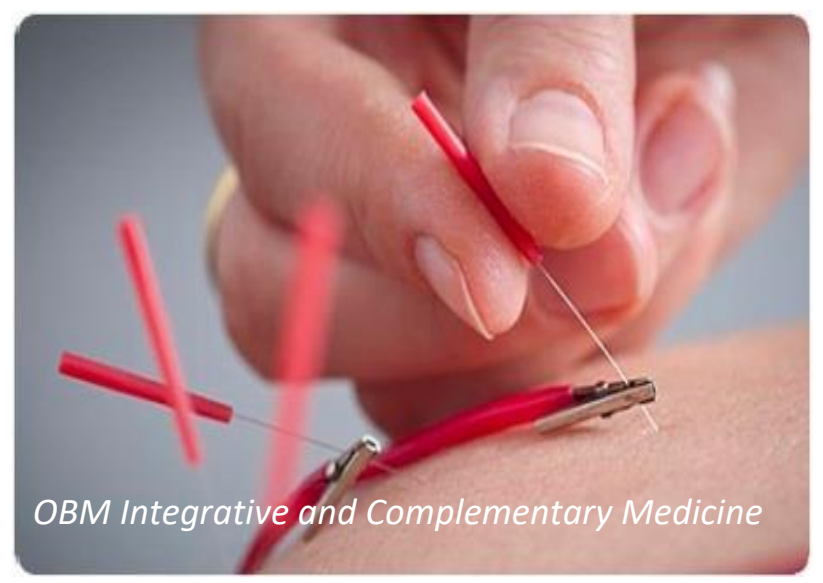

Enjoy OBM Integrative and Complementary Medicine by:

1. Submitting a manuscript

2. Joining in volunteer reviewer bank

3. Joining Editorial Board

4. Guest editing a special issue

For more details, please visit: http://www.lidsen.com/journals/icm 